\title{
Aplicación líquida edáfica y foliar de manganeso en espinaca (Spinacia oleraceae L.) cultivada en sustrato vermicompost
}

\section{Foliar and soil application of manganese in spinach (Spinacia oleraceae L.) grown in a vermicompost substrate}

ANDRÉS I. PRATO', 3

Plántulas de espinaca híbrido

Panther.

Foto: A.I. Prato

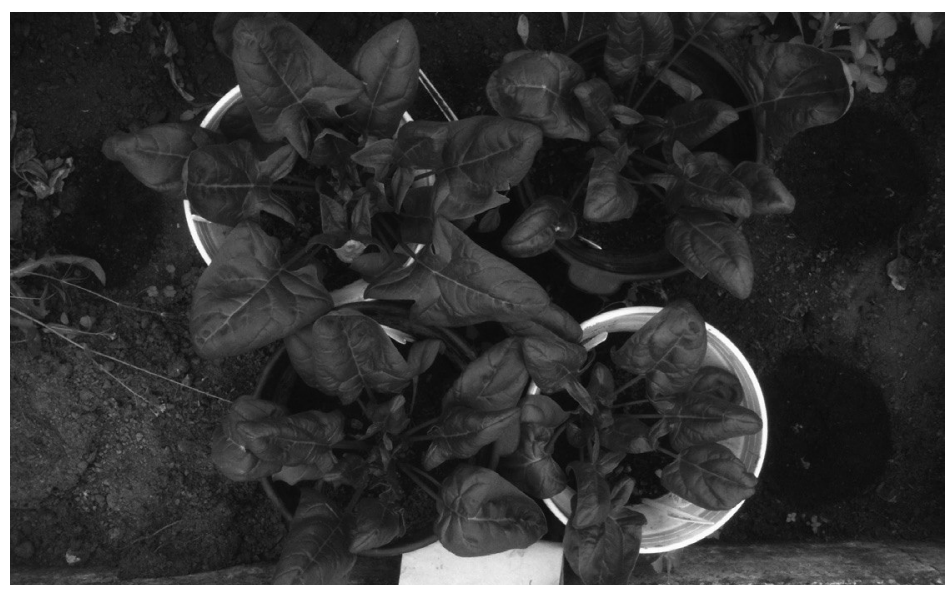

\section{RESUMEN}

En la agricultura orgánica de hortalizas, el uso de sustratos a base de vermicompost es una alternativa cada vez con mayor aceptación. La fertilización eficiente de manganeso (Mn) requiere manejos agronómicos diferenciados dadas las complejas variables de absorción y disponibilidad de este micronutriente en el suelo. Nuevos productos de fertilizantes deben ser estudiados extensamente. Se evaluó en condiciones de invernadero, el efecto de la fertilización de Mn en espinaca, híbrido Panther, empleando dos fuentes -sulfato y gluconato- en aplicación líquida edáfica y foliar, a una concentración de $50 \mathrm{mg} \mathrm{L}^{-1}\left(1,5 \mathrm{~kg} \mathrm{ha}^{-1}\right)$, y sin la aplicación del micronutriente. La fertilización fue parcializada en 10 aplicaciones. Plántulas con 15 días desde germinación fueron trasplantadas en materas de 2 L en un sustrato vermicompost. Desde los 12 días después de iniciar los tratamientos, las plantas evidenciaron efecto según la fuente y método de aplicación para la masa seca aérea y área foliar. Al final del periodo de estudio, la fuente de $\mathrm{MnSO}_{4}$ edáfico presentó la mejor respuesta, con mayor acumulación de masa seca aérea, número de hojas y altura planta frente a las plantas control, 86,7; 56,8 y 28,0\%, respectivamente. Tanto la aplicación foliar y edáfica del gluconato de Mn resultaron en valores próximos, sin síntomas visibles de toxicidad a la dosis evaluada. Este producto podría llegar a ser una opción viable de fertilización de Mn en espinaca.

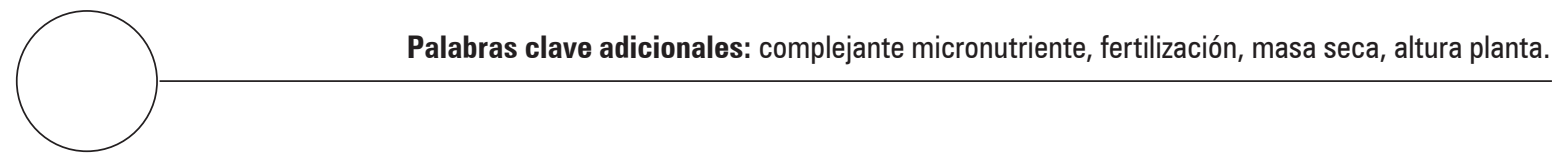

\footnotetext{
Faculdade de Agronomia, Programa Pósgraduação em Fitotecnia, Departamento de Horticultura e Silvicultura, Universidade Federal do Rio Grande do Sul, Porto Alegre (Brasil).

2 Facultad de Ciencias Agrarias, Departamento de Agronomía, Universidad Nacional de Colombia, Bogotá (Colombia).

3. Autor para correspondencia. aipratos@unal.edu.co
} 


\section{ABSTRACT}

In the organic farming of vegetables, the use of vermicompost-based substrates is an alternative with increasing acceptance. Efficient fertilization with manganese $(\mathrm{Mn})$ requires different agricultural management variables given the complex variables of absorption and availability of this micronutrient in the soil. New fertilizer products must be studied extensively. The effects of $\mathrm{Mn}$ fertilization on spinach were evaluated under greenhouse conditions using two sources - sulfate and gluconate - in liquid foliar and soil applications at a concentration of $50 \mathrm{mg} \mathrm{L}^{-1}\left(1.5 \mathrm{~kg} \mathrm{ha}^{-1}\right)$ and without the application of this micronutrient. The fertilization was separated into ten applications. Fifteen days after germination, seedlings were transplanted into $2 \mathrm{~L}$ pots filled with a vermicompost substrate. Starting at 12 days after the beginning of the treatment, the plants showed effects that depended on the source and method of the application for the aerial dry mass and leaf area. By the end of the study period, the edaphic source of $\mathrm{MnSO}_{4}$ presented the best response with a higher dry matter, number of leaves and plant height when compared to the control plants, higher by 86.7, 56.8 and $28.0 \%$, respectively. Both of the foliar and soil applications of $\mathrm{Mn}$ gluconate resulted in similar values, without symptoms of toxicity for the employed dose. This product could become a feasible option for $\mathrm{Mn}$ fertilization in spinach.

Additional key words: complex micronutrient, fertilization, dry mass, plant height.

La espinaca (Spinacia oleracea L.) se cultiva desde hace siglos en Europa y Asia. China representa el país de mayor producción y consumo, seguido de Estados Unidos y Japón (Faostat, 2013). En Colombia, el cultivo tiene más de 40 años, con las provincias del occidente y centro de la Sabana de Bogotá representando el 85\% del área nacional y con cerca de 200 ha para 2008 (Agronet, 2014).

El Mn es un micronutriente que está involucrado en la actividad de enzimas de la fotosíntesis, e interviene en la síntesis de clorofila (Taiz y Zeiger, 2006). Este elemento forma parte del complejo proteico involucrado en la fotolisis de agua en membranas de cloroplastos (Mousavi et al., 2009). En el suelo se le halla en estado intercambiable, acomplejado con sustancias orgánicas o asociado a óxidos, de esa interacción resulta su disponibilidad (Marschner, 2012).

Las deficiencias de Mn, Zn y Cu en los cultivos son tratadas normalmente con productos basa- dos en sales de sulfato, pero también se utilizan complejos y quelatos (EDTA, DTPA), o análogos. Los agentes complejantes (lignosulfonatos, heptagluconatos, humatos, gluconatos, citratos y aminoácidos) son una nueva tecnología en expansión para la agricultura (López-Rayo et al., 2011). Además, poseen la ventaja de tener un menor impacto ambiental y de toxicidad que causan las sales simples (López-Rayo et al., 2011; López-Rayo et al., 2014). Tienen como objetivo mantener los elementos complejados en forma soluble. Los gluconatos fueron incorporados hace poco a la legislación de algunos países y a pesar que normalmente son de origen natural presentan moléculas discretas. Los complejos que forman son de baja estabilidad (Lucena, 2009).

Las aplicaciones de $\mathrm{Mn}$ suelen realizarse en banda subsuperficial y al follaje, siendo el sulfato de manganeso $\left(\mathrm{MnSO}_{4}\right)$ la fuente más empleada (Papadakis et al., 2005). La baja dis- 
ponibilidad del Mn para las plantas se debe en gran medida a la formación de óxidos insolubles $\mathrm{Mn}^{\mathrm{III}}$ y $\mathrm{Mn}^{\mathrm{IV}}$, constituyendo la fracción dominante del Mn en los suelos (Binner y Schenk, 2013). También, puede ser lavado en suelos ácidos o de textura arenosa o fijados por óxidos en suelos orgánicos (Gong et al., 2010). En otros casos, para suelos con bajo contenido nativo del $\mathrm{Mn}$, calcáreos y orgánicos se recomienda fertilizaciones foliares con quelatos de Mn y así reducir su inmovilización (Lucena, 2009). La fuente orgánica (Mn-EDTA) puede ser ineficiente después de ser aplicado al suelo porque el $\mathrm{Mn}$ es rápidamente sustituido en el quelato por $\mathrm{Fe}, \mathrm{Cu}$ o Zn (Papadakis et al., 2005).

En las regiones de la Sabana de Bogotá donde se cultiva la espinaca, la deficiencia y baja disponibilidad de Mn se hace crítica debido al uso intensivo de los suelos, aplicación constante de NPK, baja tasa de restitución de micronutrientes y manejo inadecuado de enmiendas (Gómez et al., 2006).

Ante ese panorama es válido el empleo de sustratos que además de ofrecer diversas alternativas de uso, constituyen una tendencia creciente en la producción orgánica para varios cultivos hortícolas. Los vermicompost son sustratos orgánicos parcialmente degradados y estabilizados a través de la acción descomponedora (natural o antropogénica) de lombrices y microorganismos. Son ampliamente utilizados en invernaderos y viveros debido a que mejora la capacidad de almacenamiento de agua, mineralización del $\mathrm{N}, \mathrm{P}$ y K, regula favorablemente el $\mathrm{pH}$ y fomenta la actividad microbiana (Duran y Henríquez, 2007; Hashemimajdm et al., 2004). La dificultad de la fertilización del Mn en suelos orgánicos, indica que para este tipo de sustratos, el manejo de la fuente y método de aplicación específicos deban ser examinadas.

El objetivo de este experimento fue evaluar el efecto del $\mathrm{Mn}$ aplicado de forma foliar y edáfica líquida con gluconato y sulfato de $\mathrm{Mn}$ para la espinaca cultivada en un sustrato vermicompost.

\section{MATERIALES Y MÉTODOS}

El estudio fue conducido entre los meses de abril a mayo de 2012, en los invernaderos de la Facultad de Ciencias Agrarias de la Universidad Nacional de Colombia, sede Bogotá (2.556 msnm, temperatura promedio interna de $20^{\circ} \mathrm{C}$ y humedad relativa de 80\%). Plántulas de espinaca (Spinacia oleraceae L.) híbrido Panther con 2 semanas después de germinación fueron trasplantadas al lugar del experimento para iniciar los tratamientos, y dispuestas individualmente en materas de polietileno con capacidad de 2 L. Se usó un sustrato orgánico comercial a base de humus vermicompost y producido de residuos de cultivo de papa (Solanum tuberosum L.).

A partir de la solución Hoagland y Arnon se realizó fertirrigación manual, de acuerdo con los requerimientos nutricionales del cultivo de espinaca. La fertirrigación fue realizada dos veces por semana con un volumen de $160 \mathrm{~mL}$, totalizando 10 aplicaciones (tabla 1). Adicionalmente, el volumen de agua de riego a cada $2 \mathrm{~d}$, se calculó previamente mediante la diferencia de peso de las materas y relacionado con la tasa de evapotranspiración diaria del cultivo. La CE y pH en la solución nutritiva en el momento de la aplicación fue de $1,7 \mathrm{dS} \mathrm{m}^{-1}$ y $5,7 \pm 0,2$, respectivamente. Para los tratamientos foliares de Mn se empleó una bomba manual con descarga aproximada de 10 $\mathrm{mL} /$ matera en cada aplicación. Estas se realizaron separadamente del fertirriego y diluyendo la concentración calculada en agua destilada. Fue realizada dos veces por semana, totalizando 10 aplicaciones. En el caso de los tratamientos edáficos de $\mathrm{Mn}$, se realizó en conjunto con la solución preparada de los otros nutrientes.

Se implementó un diseño completamente al azar, con cinco tratamientos y 16 repeticiones, llevándose a cabo cuatro muestreos destructivos a los 14, 28, 35, 42 d después de trasplante (ddt). Se seleccionaron cuatro plantas al azar por tratamiento. En aquellos donde se aplicó $\mathrm{Mn}$, la concentración fue $50 \mathrm{mg} \mathrm{L}^{-1}\left(1,5 \mathrm{~kg} \mathrm{ha}^{-1}\right)$ y caracterizados así: fertilización sin $\mathrm{Mn}$ (control), $\mathrm{MnSO}_{4}$ 
Tabla 1. Concentración de elementos minerales en la solución madre.

\begin{tabular}{|c|l|l|}
\hline Elemento & \multicolumn{1}{|c|}{$\mathrm{mg} \mathrm{L}^{-1}$} & \multicolumn{1}{c|}{ Fuente } \\
\hline $\mathrm{N}$ & 170 & $\mathrm{Ca}\left(\mathrm{NO}_{3}\right)_{2} 4 \mathrm{H}_{2} \mathrm{O}$ \\
\hline $\mathrm{P}$ & 40 & $\mathrm{KH}_{2} \mathrm{PO}_{4}$ \\
\hline $\mathrm{K}$ & 200 & $\mathrm{KH}_{2} \mathrm{PO}_{4} ; \mathrm{KNO}_{3}$ \\
\hline $\mathrm{Ca}$ & 125 & $\mathrm{Ca}_{2}\left(\mathrm{NO}_{3}\right)_{2} 4 \mathrm{H}_{2} \mathrm{O}$ \\
\hline $\mathrm{Mg}$ & 35 & $\mathrm{MgSO}_{4} \mathrm{H}_{2} \mathrm{O}$ \\
\hline $\mathrm{S}$ & 45 & $\mathrm{MgSO}_{4} 7 \mathrm{H}_{2} \mathrm{O}$ \\
\hline $\mathrm{Zn}$ & 1 & $\mathrm{ZnSO}_{4}$ \\
\hline $\mathrm{B}$ & 1 & $\mathrm{Borato}$ \\
\hline $\mathrm{Cu}$ & 0,5 & $\mathrm{CuSO}_{4}$ \\
\hline $\mathrm{Mn}$ & 1 & $\mathrm{MnSO}_{4}$ \\
\hline Fe & 2 & $\mathrm{Fe}_{2}-\mathrm{EDTA}$ \\
\hline Mo & 0,5 & $\mathrm{H}_{3} \mathrm{MoO}_{4}$ \\
\hline *ácido cítrico anhidro & & $\mathrm{C}_{6} \mathrm{H}_{8} \mathrm{O}_{7}$ \\
\hline *EDTA sal sódica & & $\mathrm{C}_{10} \mathrm{H}_{14} \mathrm{NN}_{2} \mathrm{Na}_{2} \mathrm{O}_{8} \cdot 2 \mathrm{H}_{2} \mathrm{O}$ \\
\hline
\end{tabular}

${ }^{*}$ Acidificante y complejante usados en la preparación de la solución madre.

foliar (Mn-fol), $\mathrm{MnSO}_{4}$ edáfico (Mn-eda), gluconato de $\mathrm{Mn}$ foliar (Glu-fol) y gluconato $\mathrm{Mn}$ edáfico (Glu-eda).

Las fuentes de $\mathrm{Mn}$ empleadas fueron $\mathrm{MnSO}_{4}$ granulado (30\% Mn) y gluconato de $\mathrm{Mn}$ líquido $(10 \% \mathrm{Mn}, \mathrm{pH}=6,3)$. Se determinaron las variables área foliar (planímetro Li-3100; LI-COR, Lincoln, NE), masa seca aérea (hojas, peciolos y tallo en estufa de secado a $70^{\circ} \mathrm{C}$ por $72 \mathrm{~h}$, posterior pesaje en balanza analítica), número de hojas (más de $50 \%$ expandidas), longitud de la parte aérea (desde el cuello del tallo hasta el ápice de la lámina foliar más larga) y el índice de clorofila foliar (lectura SPAD, con el clorofilómetro SPAD 502 plus, Minolta, Tokio) tomando tres repeticiones por planta en cada tratamiento sobre la cara abaxial de la hoja

Los datos fueron sometidos a análisis de varianza. Los valores de área foliar y masa seca aérea fueron transformados mediante $\log _{\mathrm{x}} \mathrm{y} \log _{\mathrm{x}+1}$, respecti- vamente, para homogeneidad de varianzas y normalidad de datos, previa prueba Levene y Shapiro-Wilk. Cuando existió significancia, las medias fueron separadas usando la prueba de comparación de medias Tukey $(P \leq 0,05)$. El análisis estadístico mediante el programa SAS ${ }^{\circledR}$ versión 9.2

\section{RESULTADOS Y DISCUSIÓN}

De acuerdo al análisis químico antes de iniciar los tratamientos (tabla 2), el sustrato usado presentó altos contenidos de $\mathrm{Mn}\left(566 \mathrm{mg} \mathrm{kg}^{-1}\right)$. También, fue alta la materia orgánica (22\%), N (1,65\%) y el carbono orgánico oxidable $(12,7 \%)$, pero dentro de los rangos normales para abonos orgánicos (Hashemimajd et al., 2004). Resultados similares fueron encontrados para vermicompost producidos a partir de diferentes residuos, y en la cual todos los tratamientos presentaron $\mathrm{pH}$ superior a 7 (Duran y Henríquez, 2007). Valores ligeramente básicos son de esperar, porque las lombri- 
Tabla 2. Características químicas del sustrato orgánico empleado.

\begin{tabular}{|c|c|}
\hline Parámetro & Valor \\
\hline $\mathrm{pH}^{*}$ & 6,2 \\
\hline Relación C/N & 7,70 \\
\hline Materia orgánica (\%) a & 22 \\
\hline Carbono orgánico (\%) a & 12,7 \\
\hline Cenizas $(\%)^{b}$ & 60,4 \\
\hline $\mathrm{N}$ total $(\%)^{\mathrm{c}}$ & 1,65 \\
\hline P disponible (\%) ${ }^{d}$ & 1,29 \\
\hline K intercambiable (\%) ${ }^{d}$ & 1,30 \\
\hline Mg intercambiable (\%) d & 0,77 \\
\hline Ca intercambiable (\%) d & 3,35 \\
\hline $\operatorname{Mn}\left(\mathrm{mg} \mathrm{kg}^{-1}\right)^{\mathrm{e}}$ & 566 \\
\hline
\end{tabular}

*Según fabricante. Método/extractantes usado para la determinación: Walkley-Black (a), calcinación $475^{\circ} \mathrm{C}$ (b), micro-Kjeldahl (c), molibdato y vanadato aluminio (d), espectrofotometría absorción atómica (e).

ces secretan carbonato cálcico y producen una digestión alcalina.

\section{Masa seca aérea}

Hubo un cambio significativo de masa seca aérea desde los $12 \mathrm{ddt}$ hasta los $42 \mathrm{ddt}$ (figura 1). A los 12 ddt la fuente de $\mathrm{MnSO}_{4}$ fue superior al control y gluconato de $\mathrm{Mn}(P \leq 0,05)$, independiente del método de aplicación.

Para los $28 \mathrm{ddt}$, el control solo fue inferior del tratamiento Mn-eda $(P \leq 0,01)$. Sin embargo, en el último muestreo, $42 \mathrm{ddt}$, el control (3,8 g/ planta) obtuvo la menor acumulación de masa seca (MS), siendo un 86,7\% inferior del Mn-eda $(7,1 \mathrm{~g} /$ planta), tratamiento con la mejor respuesta $(P \leq 0,01)$. La aplicación edáfica $(5,6 \mathrm{~g} /$ planta $)$ y foliar (5,4 g/planta) de gluconato de $\mathrm{Mn}$ no difirió con la aplicación foliar de $\mathrm{MnSO}_{4}$ (5,3 g/ planta). En general, los complejantes de micronutrientes tienen mejor estabilidad en el suelo que los quelatos, por lo que su principal vía de actuación es en disolución nutritiva o por aplicación foliar (Lucena, 2009). Se verificó un incremento notable de la MS para el Glum-fol $(138,1 \%)$ y en menor medida del Mn-fol (65,6\%), a los $42 \mathrm{ddt}$ con relación a los $35 \mathrm{ddt}$. Los resultados indicarían una buena capacidad de penetración foliar de estas dos fuentes de $\mathrm{Mn}$ en espinaca. El efecto de la fertilización foliar depende de la velocidad de absorción del nutriente por las hojas y su translocación en la planta (Boaretto et al., 2003).

La baja acumulación de MS aérea para el control puede ser explicada porque la falta del Mn limita la eficiencia de la fotosíntesis (transporte de electrones y sistema de evolución de $\mathrm{O}_{2}$ ) y síntesis de carbohidratos, por tanto del crecimiento vegetal $y$ los rendimientos en los cultivos (Taiz y Zeiger, 2006). Así mismo, inhibe el metabolismo del N, la síntesis de lípidos y metabolitos secundarios, como el ácido giberélico, isoprenoides, precursores de auxinas, y otros cofactores promotores de crecimiento en las plantas (Malavolta et al., 1997).

Contenidos menores de $500 \mathrm{mg} \mathrm{kg}^{-1}$ de $\mathrm{Mn}$ activo (intercambiable + fácilmente reducido) en los sustratos deben ser garantizados para prevenir reacciones tóxicas en las plantas (Binner y Schenk, 2013). Ese valor está por debajo del sustrato vermicompost usado (566 $\left.\mathrm{mg} \mathrm{kg}^{-1}\right)$. En suelos con alta materia orgánica y $\mathrm{pH}$ neutro a alto, se presenta baja disponibilidad, como probablemente ocurrió. Esto sucede porque el $\mathrm{Mn}^{2+}$ soluble puede ser oxidado a $\mathrm{Mn}^{3+}$ y acomplejado fuertemente a la materia orgánica o a $\mathrm{MnO}_{2}$ (Gong et al., 2010).

\section{Área foliar}

Se comprobó un efecto positivo sobre el área foliar de las fuentes y métodos de aplicación del Mn (figura 2). A los $12 \mathrm{ddt}$, con tres aplicaciones del micronutriente, ya fue evidente 


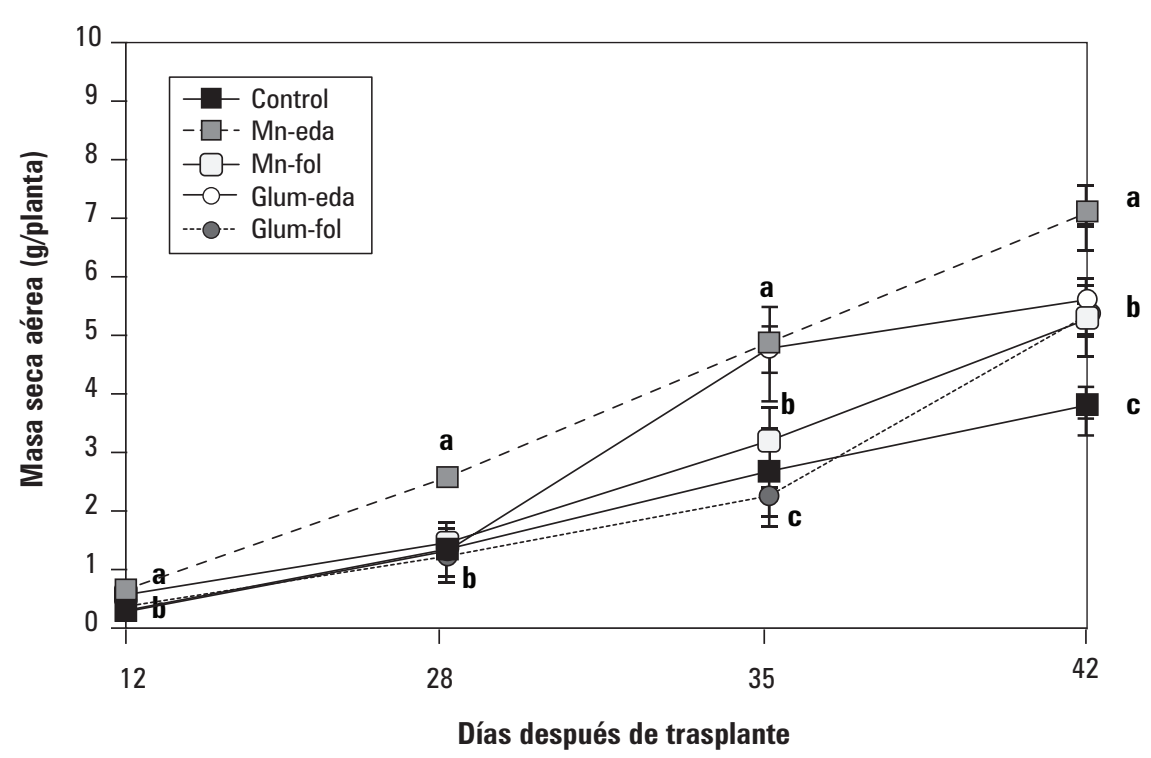

Figura 1. Efecto de la aplicación edáfica y foliar con dos fuentes comerciales de Mn en espinaca en la masa seca aérea. Promedios con letras distintas indican diferencia significativa según la prueba de Tukey $(P \leq 0,05)$. Las barras de error indican desviación estándar $(n=4)$.

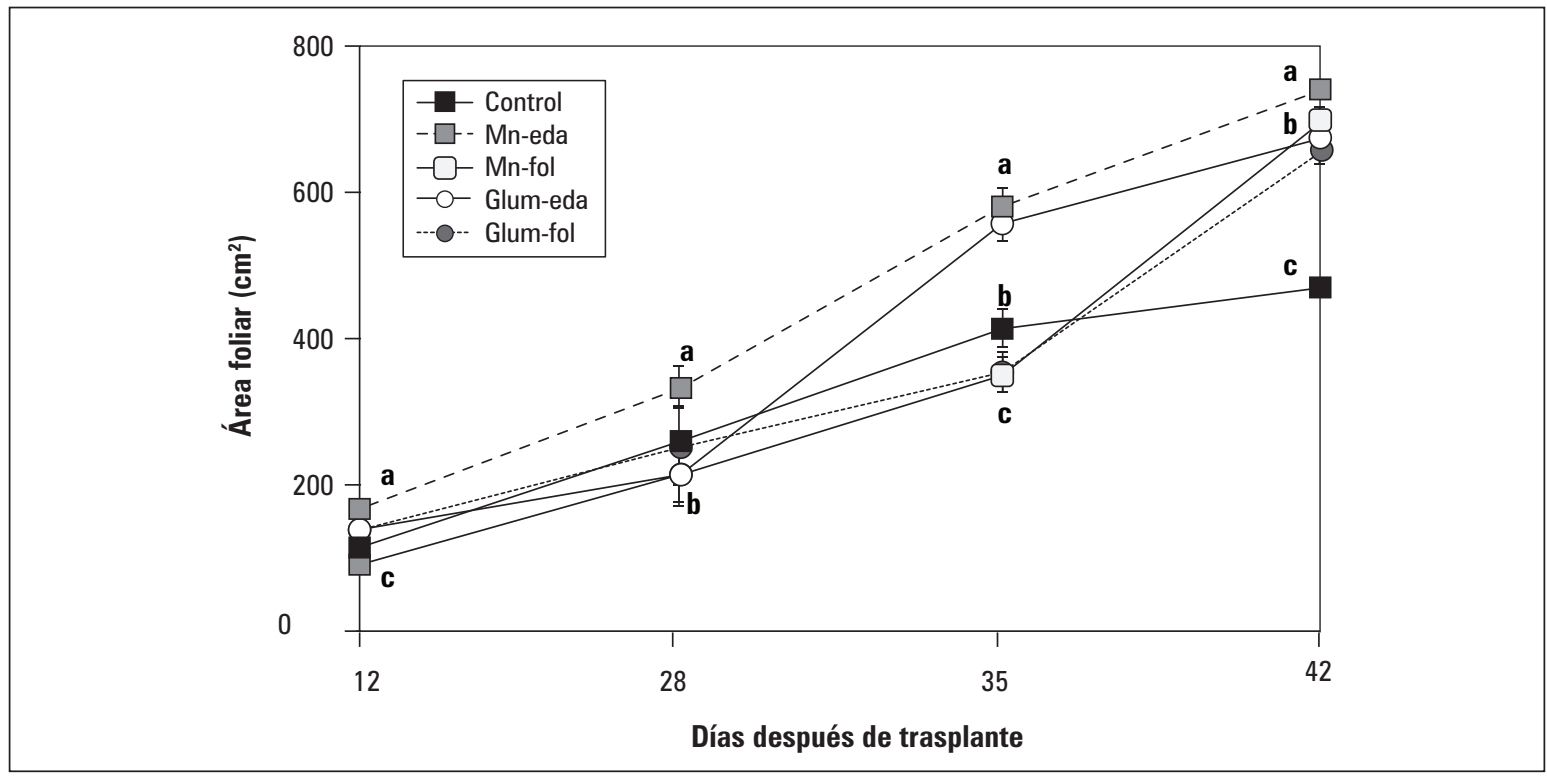

Figura 2. Efecto de la aplicación edáfica y foliar con dos fuentes comerciales de Mn en espinaca para el área foliar. Promedios con letras distintas indican diferencia significativa según la prueba de Tukey $(P \leq 0,05)$. Las barras de error indican desviación estándar $(n=4)$.

dicho efecto $(P \leq 0,001)$, probablemente a una absorción eficiente tanto por las hojas como por las raíces de espinaca. El tratamiento Glumeda tuvo el menor valor $\left(90,9 \mathrm{~cm}^{2}\right)$, mientras la aplicación foliar del gluconato de Mn exhibió la respuesta más alta $\left(138,3 \mathrm{~cm}^{2}\right)$. A partir de los
$28 \mathrm{ddt}$, independiente del método de aplicación, el Mn-eda resultó en el valor superior. Para dos portainjertos de citrus que recibieron aplicación foliar de $\mathrm{MnSO}_{4}$, se concluyó la translocación del Mn desde las hojas irrigadas a aquellas que no lo fueron (Papadakis et al., 2007). Si para la 
espinaca el Mn es también relativamente móvil, estudios más detallados son necesarios.

Para los $42 \mathrm{ddt}$, la respuesta más alta fue con Mn-eda $\left(741,2 \mathrm{~cm}^{2}\right)$, seguida del tratamiento Mn-fol $\left(699,2 \mathrm{~cm}^{2}\right)$, sin diferencia estadística. Se presentó un aumento por efecto del manejo de $\mathrm{Mn}$ del área foliar en $47 \%$ respecto al control, que tuvo el menor valor $\left(470,2 \mathrm{~cm}^{2}\right)$. Según Lucena (2009) los agentes complejantes de Mn son menos estables en el suelo, razón por la cual son usados en aplicaciones foliares. No obstante, la forma de aplicación del gluconato de Mn no fue relevante para el aérea foliar en este experimento, aunque fueron inferiores con el $\mathrm{MnSO}_{4}$. El sulfato o quelato de $\mathrm{Mn}$ son muy solubles en agua $y$ usados ampliamente en aplicaciones foliares por su eficiencia (Papadakis et al., 2007), hecho verificado en el experimento.

Aplicaciones foliares de $0,6 \%$ de $\mathrm{MnSO}_{4}$ en granado (Punica gramatum L.) incrementaron 24,2\% el área foliar más que las plantas controles, recomendando su uso por mejorar los rendimientos, 16,4\% (Hasani et al., 2012). Además, en plantas de espinaca tratadas con $40 \mathrm{~mL} \mathrm{~L}^{-1}$ cultivadas flotando sobre de una solución nutritiva completa desarrollaron un área foliar y masa seca total superior a las plantas controles, 35,2 y 21,9\%, respectivamente (Casierra et al., 2012). Esa mayor área foliar por acción del Mn fue justificado en Citrus volkameriana por un aumento en el número de cloroplastos, consecuentemente en la capacidad fotosintética (Papadakis et al., 2005). Si ese mecanismo también ocurre en espinaca, otros estudios son necesarios para comprobarlo.

Es importante identificar la estructura química y la composición de estos nuevos productos de complejantes, y así establecer los manejos adecuados para su empleo en la fertilización. Por ejemplo, López-Rayo et al. (2014) mediante resonancia nuclear magnética registraron impurezas desconocidas en varios quelatos y complejos (gluconato y heptogluconato) de $\mathrm{Mn}, \mathrm{Cu}$ y Fe no cuantificadas por el fabricante.

\section{Índice de clorofila foliar}

Hubo diferencias para las lecturas SPAD (verdor de las hojas) entre los tratamientos a los $42 \mathrm{ddt}(P \leq 0,01)$. El tratamiento Mn-fol $(69,1)$ tuvo el valor más alto, pero solo significativo con respecto al Glum-eda $(P \leq 0,01)$ y el control $(P \leq 0,05)$ (tabla 3$)$. Existe una respuesta favorable del Mn en la activación del proceso fotosintético para esta especie debido a su función fisiológica en la reacción de Hill del FSII (Gong et al., 2010).

En otros estudios, Sabin et al. (2011) no evidenciaron diferencias empleando fuentes (producto comercial de varios micronutrientes, quelato y sulfato de $\mathrm{Mn}$ ) y dosis de $\mathrm{Mn}$ (hasta $10 \mathrm{~kg} \mathrm{ha}^{-1}$ ) aplicados caña de azúcar para las lecturas del clorofilómetro.

A partir de los resultados del índice de clorofila foliar (lecturas SPAD), área foliar y ausencia de manchas necróticas en los bordes y tonalidades rojizas o marrones en la nervadura de las hojas, parece no haber un efecto toxico de las fuentes usadas a la dosis de $50 \mathrm{mg} \mathrm{L}^{-1}$ de $\mathrm{Mn}$. No se apreciaron síntomas visuales de deficiencia (manchas amarillas intervenales en hojas jóvenes). Los resultados están en concordancia con los obtenidos por Casierra et al. (2012) a una dosis de $40 \mathrm{mg} \mathrm{L}^{-1} \mathrm{Mn}$ en plantas de espinaca, donde a $80 \mathrm{mg} \mathrm{L}^{-1}$ si resultó en toxicidad.

Sin embargo, la toxicidad por Mn es tal vez el factor más importante después del aluminio para plantas en suelos ácidos (Mou et al., 2011). Tales características edáficas son típicas en las regiones de la Sabana de Bogotá. En suelos andisoles y con más de $60 \mathrm{mg} \mathrm{kg}^{-1}$ de $\mathrm{Mn}$, la causa principal de las manchas amarillas intervenales en cultivos de espinaca fue la deficiencia del micronutriente, asociado a la fuerte adsorción de este suelo (Sakata y Yoshida, 2007). Los síntomas de deficiencia por Mn en plantas pueden ocurrir muy lentamente, por lo que el productor no daría cuenta de la situación con rapidez. 


\begin{tabular}{|l|c|c|c|}
\multicolumn{1}{r|}{ Tratamiento } & Índice clorofila foliar (SPAD) & Número de hojas & Longitud de parte aérea (cm) \\
\hline Control & $63,75 \pm 2,5 \mathrm{~b}$ & $18,50 \pm 1,2 \mathrm{~d}$ & $25,00 \pm 2,1 \mathrm{c}$ \\
\hline $\mathrm{MnSO}_{4}$ edáfico & $65,53 \pm 3,6 \mathrm{ab}$ & $29,00 \pm 1,1 \mathrm{a}$ & $32,00 \pm 1,4 \mathrm{a}$ \\
\hline $\mathrm{MnSO}_{4}$ foliar & $69,10 \pm 2,8 \mathrm{a}$ & $25,00 \pm 1,4 \mathrm{~b}$ & $29,00 \pm 1,8 \mathrm{ab}$ \\
\hline Gluconato de Mn edáfico & $60,43 \pm 3,5 \mathrm{~b}$ & $21,75 \pm 1,2 \mathrm{c}$ & $27,25 \pm 1,8 \mathrm{bc}$ \\
\hline Gluconato de Mn foliar & $65,25 \pm 4,0 \mathrm{ab}$ & $26,50 \pm 1,2 \mathrm{ab}$ & $29,25 \pm 2,2 \mathrm{ab}$ \\
\hline Promedio & 64,83 & 24,15 & 28,50 \\
\hline CV(\%) & 4,85 & 17,00 & 9,09 \\
\hline
\end{tabular}

Tabla 3. Respuesta de espinaca para las variables fisiológicas evaluadas a los $\mathbf{4 2}$ ddt. Medias \pm desviación estándar $(n=4)$. Promedios con letras distintas indican diferencia significativa según la prueba de Tukey $(P \leq 0,05)$.

De esta manera resalta la importancia que tiene el micronutriente en el contexto de la producción regional en Colombia.

\section{Número de hojas y longitud de parte aérea}

La respuesta en el número de hojas dependió de los tratamientos $(P \leq 0,0001)$, siendo $\mathrm{Mn}$-eda superior (29) seguido de las fuentes de $\mathrm{Mn}$ con aplicación foliar $(25,8)$. Las plantas control alcanzaron en promedio 18,5 hojas, confirmando un menor desarrolló vegetativo (tabla 3). Dosis de 6 y $9 \mathrm{mg} \mathrm{kg}^{-1} \mathrm{Mn}$-EDTA, incrementaron el número de vainas por planta en fríjol, 31,2 y $94,2 \%$, respectivamente, y siendo más eficientes en aplicación foliar (Ozbahce y Zengin, 2011).

La longitud de la parte aérea fue afectada por el manejo de $\mathrm{Mn}(P \leq 0,01)$. Las plantas del tratamiento control exhibieron una menor altura, $15,0 \%$ ó 4,4 cm menos, frente a aquellas fertilizadas (tabla 3). Simoglou y Dordas (2006) observaron diferencia significativa para la altura de plantas de trigo 'duro' (Triticum turgidum L. subsp. durum Desf.) cuando recibieron aplicación foliar de $\mathrm{MnSO}_{4}$, siendo $3 \mathrm{~cm}$ superior a las plantas control. Igualmente, en fríjol común (Phaseolus vulgaris) con dos aplicaciones foliares de Mn ocurrió un aumento en la altura del 34\% a la dosis de $300 \mathrm{~g} \mathrm{ha}^{-1} \mathrm{Mn}$, frente a la ausencia de fertilización (Teixeira, 2004).

La literatura reporta variación en el efecto según el tipo de fuente usada de Mn. Laurie et al. (1995) identificaron mejor absorción de Mn en avena cuando era acomplejado entre un 0 a 45\% con EDTA en la solución nutritiva. Rodríguez et al. (2010) concluyeron que quelatos sintéticos fueron más eficientes que complejos naturales para corregir clorosis férrica cuando se aplicó en fertirrigación o al follaje en soya. Sabin et al. (2011), también indicaron con esa misma fuente que la aplicación al suelo proporcionó mayor número de tallos en caña de azúcar. Por otro lado, en suelos calcáreos y bajo Mn disponible, tanto quelatos como complejos mantuvieron más $\mathrm{Mn}$ en la solución del suelo que el $\mathrm{MnSO}_{4}$, siendo una mejor alternativa por fertirrigación y en aplicación al suelo (López et al., 2014). Es decir, dada la complejidad estructural de estos complejantes, los estudios sobre su eficiencia ha dado resultado disimiles.

\section{CONCLUSIONES}

El $\mathrm{MnSO}_{4}$ líquido edáfico proporciona un mayor aumento en la MS aérea, número de hojas y altu- 
ra de parte aérea en comparación a las plantas de espinaca sin fertilización con $\mathrm{Mn}$.

El gluconato de Mn podría ser una fuente de fertilización foliar o edáfica en espinaca cultivada en sustrato vermicompost, por tanto, próximos estudios deberían evaluar dosis más altas del producto.

\section{AGRADECIMIENTOS}

A los docentes del curso Fertilizantes y Fertilización, y funcionarios del Laboratorio de Fisiología de Cultivos de la Facultad de Ciencias Agrarias de la Universidad Nacional de Colombia, Bogotá, por el acompañamiento, asesoría y disponibilidad de recursos para la realización del experimento.

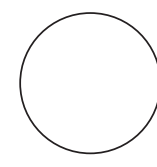

Agronet. 2014. Área y producción agrícola y pecuaria. Análisis - estadísticas. En: http://agronet.gov.co; consulta: marzo de 2014

Binner, I. y M. Schenk. 2013. Manganese in substrate clays - harmful for plants?. J. Plant. Nutr. Soil Sci. 176(5), 809-817. Doi: 10.1002/jpln.201200433

Boaretto, A., T. Muraoka y R. Boaretto. 2003. Absorção e translocação de Mn, Zn e B aplicados via foliar em citros. Laranja 24(1), 177-197.

Casierra, F., C. Ulrichs y C. Pérez. 2012. Growth of spinach plants (Spinacia oleracea L.) exposed to excess zinc and manganese. Agron. Colomb. 30(3), 344350 .

Durán, L. y C. Henríquez. 2007. Caracterización química, física y microbiológica de vermicompostes producidos a partir de cinco sustratos orgánicos. Agron. Costarr. 31(1), 41-51.

Faostat. 2013. Series cronológicas y de datos con relación a la alimentación y agricultura. FAO, Roma.

Gómez, M.I., M. López e Y. Cifuentes. 2006. El manganeso como factor positivo en la producción de papa (Solanum tuberosum L.) y arveja (Pisum tuberosum L.) en suelos del altiplano Cundiboyacense. Agron. Colomb. 24(2), 340-347.

Gong, X., Y. Wang, C. Liu, S. Wang, X. Zhao, M. Zhou, N. Li, Y. Lu y F. Hong. 2010. Effects of manganese deficiency on spectral characteristics and oxygen evolution in maize chloroplasts. Biol. Trace Elem. Res. 136(3), 372-382. Doi: 10.1007/s12011-0098549-9

Hasani, M., Z. Zamani, G. Savaghebi y R. Fatahi. 2012. Effects of zinc and manganese as foliar spray on pomegranate yield, fruit quality and leaf minerals.

\section{REFERENCIAS BIBLIOGRÁFICAS}

J. Soil Sci. Plant Nutr. 12(3), 471-480. Doi: http:// dx.doi.org/10.4067/S0718-95162012005000009

Hashemimajdm, K., M. Kalbasi, A. Golchin y H. Shariatmandari. 2004. Comparison of vermicompost and compost as potting media for growth of tomatoes. J. Plant Nutr. 27(6), 1107-1123. Doi: 10.1081/ PLN-120037538

Laurie, S., N. Tancock, S. McGrath y J. Sanders. 1995. Influence of EDTA complexation on plant uptake of manganese (II). Plant Sci. 109(2), 231-235. Doi: 10.1016/0168-9452(95)04161-M

López-Rayo, S., J. Lucena, L. Lagui y M. Cremonini. 2011. Demetalation of $\mathrm{Fe}, \mathrm{Mn}$, and $\mathrm{Cu}$ chelates and complexes: application to the NMR analysis of micronutrient fertilizers. J. Agric. Food Chem 59(24), 13110-13116. Doi: 10.1021/jf203602a

López-Rayo, S., S. Lucena y J. Lucena. 2014. Chemical properties and reactivity of manganese chelates and complexes in solution and soils. J. Plant Nutr. Soil Sci. 177(2), 189-198. Doi: 10.1002/ jpln.201300091

Lucena, J. 2009. El empleo de complejantes y quelatos en la fertilización de micronutrientes. Rev. Ceres 56(4), 527-535.

Malavolta, E., G. Vitti y S. Oliveira. 1997. Avaliação do estado nutricional das plantas: princípios e aplicações. Potafos, Piracicaba, São Paulo, Brasil.

Marschner, P. (ed.). 2012. Mineral nutrition of higher plants. $3^{\text {th }}$ ed. Academic Press, London.

Mou, D., Y. Yao, Y. Yang, Y. Zhang, C. Tian y V. Achal. 2011. Plant high tolerance to excess manganese related with root growth, manganese distribution 
and antioxidative enzyme activity in three grape cultivars. Ecotoxicol. Environ. Saf. 74(4), 776-786. Doi: 10.1016/j.ecoenv.2010.10.040

Mousavi, S., M. Shahsavari y M. Rezae. 2011. A general overview on manganese $(\mathrm{Mn})$ importance for crops production. Aust. J. Basic Appl. Sci. 5(9), 1799-1803.

Özbahçe, A. y M. Zengin. 2011. Effects of manganese fertilizers on yield and yield components of dwarf dry bean. J. Plant Nutr. 34(1), 127-139. Doi: 10.1080/01904167.2011.531604

Papadakis, I., T. Protopapadakis, I. Therios y V. Tsirakoglo. 2005. Foliar treatment of $\mathrm{Mn}$ deficient 'Washington navel' orange trees with two $\mathrm{Mn}$ sources. Sci. Hortic. 106(1), 70-75. Doi: 10.1016/j. scienta.2005.02.015

Papadakis, I., T. Sotiropoulos e I. Therios. 2007. Mobility of iron and manganese within two citrus genotypes after foliar applications of iron sulfate and manganese sulfate. J. Plant Nutr. 30(9), 1385 1396. Doi: 10.1080/01904160701555754

Rodríguez, P., L. Hernández y J. Lucena. 2010. Comparison of iron chelates and complexes supplied as fo- liar sprays and in nutrient solution to correct iron chlorosis of soybean. J. Plant Nutr. Soil Sci. 173(1), 1120-126. Doi: 10.1002/jpln.200800256

Sabin, C., S. Buzetti, K. Silva, M. Carvalho, C. Garcia y P. Mistrelo. 2011. Produtividade e desenvolvimento da cana-planta e soca em função de doses e fontes de manganês. R. Bras. Ci. Solo 35(5), 1661-1668.

Simoglou, K. y C. Dordas. 2006. Effect of foliar applied boron, manganese and zinc on tan spot in winter durum wheat. Crop Prot. 25(7), 657-663. Doi: 10.1016/j.cropro.2005.09.007

Sakata, I. y T. Yoshda. 2007. Manganese deficiency of the Andosol in Northern part of Gifu Pref. caused the yellow spot in spinach in Takayama city, Takane-cho. Jpn. J. Crop Sci. 76(2), 311-316.

Taiz, L. y E. Zeiger. 2006. Plant physiology. $4^{\text {th }}$ ed. Sinauer Associates Publishers, Sunderland, MA.

Teixeira, I., A. Borém, G. Araújo y R. Fontes. 2004. Manganese and zinc leaf application on common bean grown on a "cerrado" soil. Sci. Agric. 61(1), 77-81. Doi: 10.1590/S0103-90162004000100013 